\title{
Electron dynamics during substorm dipolarization in Mercury's magnetosphere
}

\author{
D. C. Delcourt ${ }^{1}$, K. Seki ${ }^{2}$, N. Terada ${ }^{2, *}$, and Y. Miyoshi ${ }^{2}$ \\ ${ }^{1}$ CETP-CNRS-IPSL, Saint-Maur des Fossés, France \\ ${ }^{2}$ STEL, Nagoya University, Toyokawa, Japan \\ *now at: NIICT, Tokyo and CREST-JSTA, Saitama, Japan
}

Received: 20 May 2005 - Revised: 27 September 2005 - Accepted: 28 September 2005 - Published: 30 November 2005

\begin{abstract}
We examine the nonlinear dynamics of electrons during the expansion phase of substorms at Mercury using test particle simulations. A simple model of magnetic field line dipolarization is designed by rescaling a magnetic field model of the Earth's magnetosphere. The results of the simulations demonstrate that electrons may be subjected to significant energization on the time scale (several seconds) of the magnetic field reconfiguration. In a similar manner to ions in the near-Earth's magnetosphere, it is shown that low-energy (up to several tens of $\mathrm{eV}$ ) electrons may not conserve the second adiabatic invariant during dipolarization, which leads to clusters of bouncing particles in the innermost magnetotail. On the other hand, it is found that, because of the stretching of the magnetic field lines, high-energy electrons (several keVs and above) do not behave adiabatically and possibly experience meandering (Speiser-type) motion around the midplane. We show that dipolarization of the magnetic field lines may be responsible for significant, though transient, (a few seconds) precipitation of energetic (several keVs) electrons onto the planet's surface. Prominent injections of energetic trapped electrons toward the planet are also obtained as a result of dipolarization. These injections, however, do not exhibit short-lived temporal modulations, as observed by Mariner-10, which thus appear to follow from a different mechanism than a simple convection surge.
\end{abstract}

Keywords. Magnetospheric physics (Planetary magnetospheres; Storms and substorms) - Space plasma physics (Charged particle motion and acceleration)

\section{Introduction}

Most of our knowledge about the magnetized environment of Mercury comes from three passes of Mariner-10 in 1974 and 1975. The data obtained during these passes most notably revealed that Mercury possesses an intrinsic magnetic field

Correspondence to: D. C. Delcourt

(dominique.delcourt@ cetp.ipsl.fr) that leads to the formation of a small-scale magnetosphere (e.g. Ness, 1979). Given the small magnitude of the internal field and the enhanced solar wind pressure due to proximity with the Sun, the Hermean magnetosphere is expected to be very dynamical. As a matter of fact, the Mariner-10 data recorded in March 1974 revealed that this magnetosphere may be subjected to rapid reconfigurations. During the inbound portion of this $\sim 20$-min pass, the magnetic field in the nightside sector was found to point essentially in the Sun-tail direction, whereas it abruptly turned northward after the closest approach to the planet. Interestingly enough, high-energy (several tens of keVs and above) electron injections were recorded in conjunction with this rapid change in the magnetic field orientation. Particle measurements at lower energies were unfortunately not available but, altogether, these observations are reminiscent of the features observed during dipolarization in the terrestrial magnetosphere. This suggests that the Hermean magnetosphere may exhibit substorm cycles, as well (e.g. Siscoe et al., 1975; Slavin, 2004). In addition, a clear temporal modulation with a period of $\sim 6 \mathrm{~s}$ was identified in the energetic electron injections, the origin of which remains unclear (e.g. Christon et al., 1987). It has been proposed, for instance, that these injections follow from reconnection in the mid-tail (e.g. Eraker and Simpson, 1986) or from drift echoes of energetic electrons transported into the immediate vicinity of the planet (e.g. Baker et al., 1986). In contrast to these studies, Luhmann et al. (1998) suggested that the features observed by Mariner-10 may be directly driven by rapid changes in the solar wind.

In this study, we consider the substorm interpretation framework and use 3-D test particle simulations to examine the impact of presumed dipolarization of the magnetospheric field lines on particle transport and energization. Using twodimensional simulations, Ip (1997) demonstrated that $\mathrm{keV}$ acceleration may be achieved for ions during such reconfiguration events. Here, we focus on the dynamics of electrons. To do so, we adapted a 3-D time-dependent test particle code previously developed for the Earth's magnetosphere (Delcourt et al., 1990) and rescaled it to the environment of 

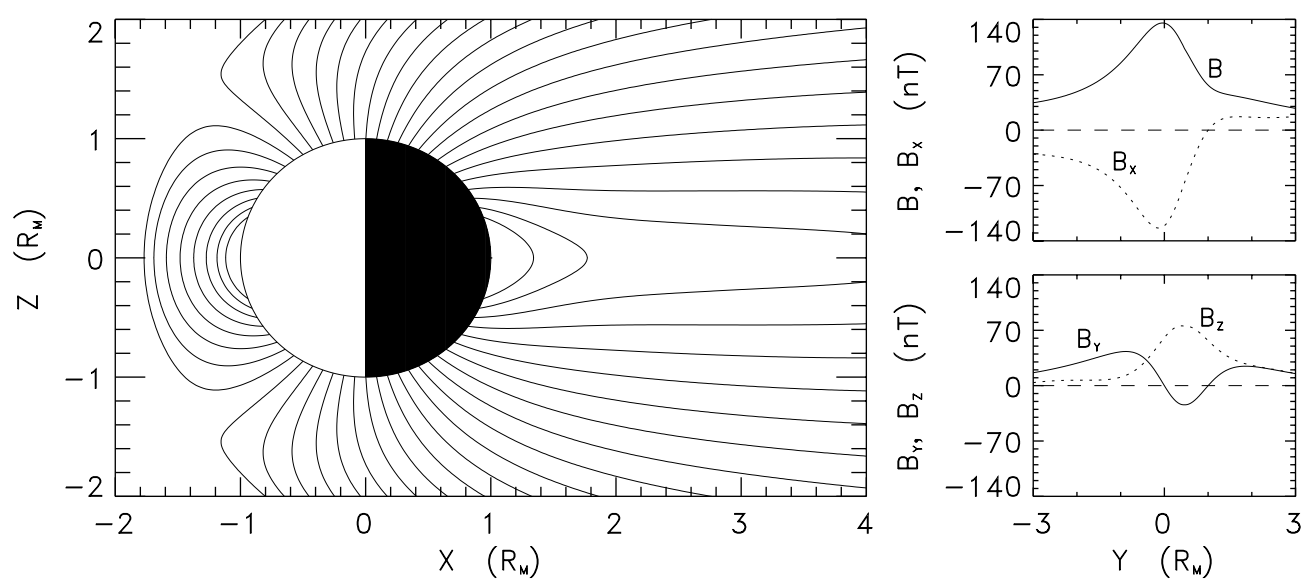

Fig. 1. (Left) Model magnetic field lines at Mercury, assuming that one Hermean radius accounts for 6 terrestrial radii in T-89. (Right) Model magnetic field variations obtained along the Mariner-10 pass in March 1974.

Mercury. We show that, because of the small spatial and temporal scales of the Hermean magnetosphere, electrons may be subjected to quite significant energization during relaxation of the magnetotail and associated convection surge toward the planet. In Sect. 2, we present the dipolarization model. In Sect. 3, we examine some characteristic features of the electron dynamics during magnetic field line reconfiguration, whereas the injection and precipitation of energetic electrons are discussed in Sect. 4.

\section{Modeling of magnetic field line dipolarization at Mer- cury}

To model the dipolarization of magnetospheric field lines at Mercury, we adopt an approach similar to that used in the study of Delcourt et al. (1990). In that study, the magnetic field line dipolarization was accounted for by considering a gradual transition within the Mead and Fairfield (1975) model, from a given level of magnetic activity to a less disturbed one. In Delcourt et al. (1990), the magnetic field at a given position $\mathbf{r}$ and a given time $t$ was obtained as:

$\mathbf{B}(\mathbf{r}, t)=\mathbf{B}_{\text {pre- }}(\mathbf{r})+f(t)\left[\mathbf{B}_{\text {post }-}(\mathbf{r})-\mathbf{B}_{\text {pre- }}(\mathbf{r})\right]$,

where $\mathbf{B}_{\text {pre }}$ and $\mathbf{B}_{\text {post }- \text { correspond to initial and final con- }}$ figurations, respectively. Also in this equation, $f(t)$ is a polynomial of degree 5 that smoothly varies between 0 at $t=0$ and 1 at $t=\tau$ (denoting by $\tau$ the time scale of the magnetic transition; see Appendix A of Delcourt et al., 1990). As for the electric field, it was considered to be the sum of two contributions. One of them is purely induced by the timevarying magnetic field, whereas the other is irrotational and accounts for plasma polarization. As discussed by Heikkila and Pellinen (1977), this latter contribution, which is built by free charges is introduced to cancel the parallel component of the electric field obtained from Faraday's law and leads to a redistribution of the electric field in the perpendicular di- rection (see Appendix B of Delcourt et al., 1990). At a given position $\mathbf{r}$ and time $t$, the electric field was thus obtained as:

$\mathbf{E}(\mathbf{r}, t)=-\frac{\partial \mathbf{A}(\mathbf{r}, t)}{\partial t}-\nabla \Phi_{p o l}(\mathbf{r}, t)$,

where $\Phi_{p o l}$ is the electric potential due to the free charges, and $\mathbf{A}$ is the instantaneous vector potential such that:

$\mathbf{A}(\mathbf{r}, t)=\mathbf{A}_{\text {pre- }}(\mathbf{r})+f(t)\left[\mathbf{A}_{\text {post }-}(\mathbf{r})-\mathbf{A}_{\text {pre- }}(\mathbf{r})\right]$,

where $\mathbf{A}_{\text {pre }}$ - and $\mathbf{A}_{\text {post - relate to initial and final configura- }}$ tions, respectively.

In the present study, we adopt a calculation technique similar to Eqs. (1)-(3) but use the model of Tsyganenko (1989) (hereinafter denoted as T-89) that covers a wider range of radial distances. In a similar way to Luhmann et al. (1998), we model the magnetosphere of Mercury by assuming that one planetary radius in this magnetosphere corresponds to 6 planetary radii in the terrestrial one. The magnetic field lines obtained after such a rescaling can be appreciated in the left panel of Fig. 1. Consistently with other models (e.g. Ip, 1997; Luhmann et al., 1998), it can be seen that the planet occupies a much wider volume of the magnetosphere than in the terrestrial case, with a subsolar point located at $\sim 1.8 \mathrm{R}_{M}$ and with cusp field lines anchored near $55^{\circ}$ latitude (note that in the following the $X$ and $Y$ axis point tailward and dawnward, respectively). Simultaneously, the right panels of Fig. 1 present the magnetic field variations obtained along the Mariner-10 pass using the present model. A fair agreement is obtained between these variations and those recorded by Mariner-10 (see, e.g. Luhmann et al., 1998) which qualitatively validates the present model.

To simulate the dipolarization of the magnetic field lines, we use the above Eqs. (1)-(3) and consider an overall transition from $K_{p}=2$ to $K_{p}=0$ within the rescaled T-89. Assuming that time scales at Mercury are $\sim 30$ times smaller than those at Earth (e.g. Russell and Walker, 1985), we also set $\tau$ to $5 \mathrm{~s}$. The reconfiguration thus obtained is comparable to 

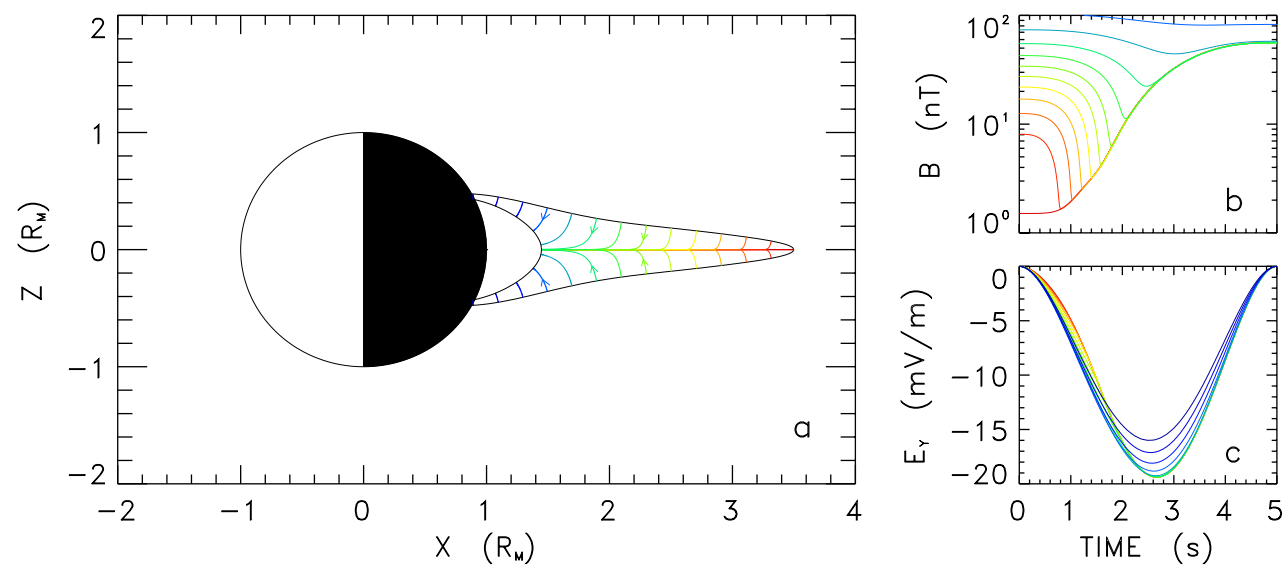

Fig. 2. Model dipolarization in the rescaled T-89: (a) initial and final magnetic field configuration, (b) magnetic field and (c) electric field variations encountered along the different paths shown in the left panel. The different colors correspond to different initial curvilinear abscissa (separated by equidistant steps of $500 \mathrm{~km}$ ) along the field line. Initial and final magnetic activity levels in T-89 are set to $K_{p}=2$ and $K_{p}=0$, respectively, whereas the dipolarization time scale is set to $5 \mathrm{~s}$.

the two-dimensional model of Ip (1997). Figure 2 displays the evolution of the magnetic field line initially intercepting the equator at $3.5 \mathrm{R}_{M}$. Black lines in this figure show the pre- and post-dipolarization configurations of the magnetic field line, whereas the color-coded ones show the temporal evolution of selected points (separated by equidistant steps of $500 \mathrm{~km}$ ) along the field line or, equivalently, the trajectories of virtual zero-energy particles that are only subjected to $\boldsymbol{E} \times \boldsymbol{B}$ drift during dipolarization. As expected, it is apparent from Fig. 2a that the magnetic field line exhibits a more dipolar shape after the magnetic transition, its equatorial intersection moving from $3.5 \mathrm{R}_{M}$ at onset down to $\sim 1.5 \mathrm{R}_{M}$ at the end of the dipolarization. As for the points located offequator, it can be seen that they are first transported toward the $Z=0$ plane and subsequently turned toward the planet. As will be seen hereinafter, such an abrupt change in the $\boldsymbol{E} \times \boldsymbol{B}$ drift orientation has significant consequences on the net parallel acceleration of the particles.

Figures $2 b$ and $2 c$ present the magnetic and electric field variations encountered along the color-coded paths in the left panel. As for the equatorial foot of the field line (path in dark red), it can be seen in Fig. 2b that the magnetic field varies from $\sim 1.5 \mathrm{nT}$ at onset up to more than $50 \mathrm{nT}$ at the end of the dipolarization. As for off-equator points, their motion toward the $Z=0$ plane first yields a sharp decrease in the $B$ magnitude, which is followed by an increase when the points turn toward the planet. In conjunction with this equatorward and inward transport, Fig. 2c displays a large duskward oriented transient electric field, with a peak magnitude of the order of $20 \mathrm{mV} / \mathrm{m}$ for the low-latitude paths and a smaller magnitude at higher latitudes.

\section{Model electron orbits during dipolarization}

As is the case in the Earth's magnetosphere, we expect the dipolarization and associated convection surge in Fig. 2 to be responsible for significant particle energization. In an early study of substorm dipolarization at Earth, Mauk (1986) demonstrated that, because the convection surge occurs on a time scale comparable to the ion bounce period, different parallel energization may be achieved depending upon bounce phase at onset, which leads to a violation of the second adiabatic invariant (viz., $\int v_{/ /} \mathrm{d} s$, where $v_{/ /}$is the parallel speed and $s$ the curvilinear abscissa along the field line), i.e. particles which intercept the equator during dipolarization are subjected to a large transient electric field and prominent energization, whereas those that do not cross the equator remain essentially unaffected. It was argued that such a differential energization is at the origin of the bouncing ion clusters frequently observed in the inner Earth's magnetosphere during substorms, as initially suggested by Quinn and McIlwain (1979). On the other hand, it was shown in Delcourt et al. (1990) that the first adiabatic invariant (the particle magnetic moment) may actually not be conserved during dipolarization, allowing for enhanced energization in the perpendicular direction. For a given relaxation time scale, such a nonadiabatic heating is preferentially obtained for heavy ions that have large cyclotron periods, a process that may be responsible for the species dependent energization observed in the storm-time ring current (e.g. Mitchell et al., 2003).

For electrons in the model presented in Fig. 2, the cyclotron period is of at most a few tens of milliseconds, so that we do not expect a violation of the first adiabatic invariant due to the time-varying fields. If such a violation of the first adiabatic invariant occurs, it is rather due to the pronounced stretching of the magnetic field lines in the mid-tail, which leads to Larmor radii comparable to, or larger than, the field variation length scale. In other words, while temporal nonadiabaticity is unlikely, spatial nonadiabaticity cannot be ruled out for electrons. As a matter of fact, making use of the adiabaticity parameter $\kappa$, introduced by Büchner and Zelenyi (1989) (defined as the square root of the minimum 


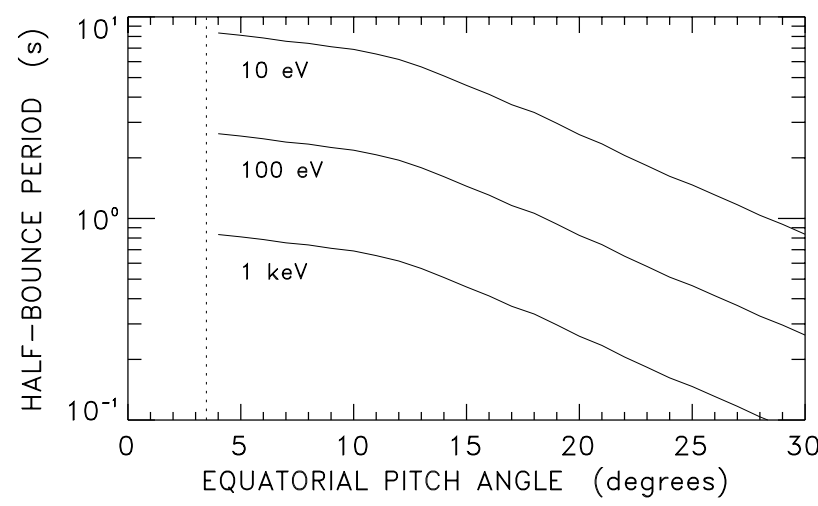

Fig. 3. Half-bounce period of electrons with different energies $(10 \mathrm{eV}, 100 \mathrm{eV}, 1 \mathrm{keV})$ along the field line intercepting the equator at $3.5 \mathrm{R}_{M}$. The half-bounce period is shown as a function of pitch angle at the equator. The vertical dotted line shows the loss cone limit.

curvature radius to maximum Larmor radius ratio), it will be seen hereinafter that electrons with energies of several keVs and above do exhibit a $\kappa$ parameter smaller than 3 , in which case the magnetic moment may not be conserved and the guiding center approximation is not valid. For this reason, test particle trajectory computations were performed here using the full equation of motion, integrated by means of a fourth-order Runge-Kutta algorithm. As in previous numerical studies, the time step of integration was adjusted to the particle gyro-period, corresponding to a change of $5^{\circ}$ degrees in gyration phase. Comparison with simulations using different time steps of integration showed that the results obtained are robust.

On the other hand, low-energy electrons may exhibit bounce periods that are comparable to, or larger than, the dipolarization time scale, so that their second adiabatic invariant may not be conserved. Figure 3 shows the halfbounce period of electrons along the magnetic field line displayed in Fig. 2, considering different energies $(10 \mathrm{eV}$, $100 \mathrm{eV}$ and $1 \mathrm{keV}$ ) and various pitch angles at the equator. It is apparent from this figure that, with an equatorial footpoint at $3.5 \mathrm{R}_{M}, 1 \mathrm{keV}$ electrons have a bounce period smaller than $1 \mathrm{~s}$. Accordingly, one does not expect a significant dependence upon the initial bounce phase during the 5-s magnetic transition. In contrast, electrons with $10 \mathrm{eV}$ energy may exhibit bounce periods of $\sim 10 \mathrm{~s}$ or above, so that different parallel energization depending upon initial conditions, is to be expected and the second adiabatic invariant is likely to be violated.

Figure 4 shows examples of electron orbits obtained in the model dipolarization displayed in Fig. 2. In Fig. 4, 10-eV test electrons were launched at $90^{\circ}$ pitch angle from different (color-coded) locations on the field line intercepting the equator at $X=3.5 \mathrm{R}_{M}$. Let us first consider the test electron with $90^{\circ}$ pitch angle at the equator (orbit in dark red). This particle behaves adiabatically while being rapidly convected down to $\sim 1.5 \mathrm{R}_{M}$. During this transport, because of magnetic moment conservation, it experiences a betatron energization with a net energy gain in proportion to the magnetic field change (by a factor 30-40; Fig. 4b). Also, in Fig. 4c, it can be seen that this test electron remains trapped at the equator, with a $90^{\circ}$ pitch angle throughout the dipolarization process.

For the test electron initialized immediately above the midplane (orbit in light red), Fig. 4 also displays a substantial energization (by about a factor 10) while the particle keeps bouncing in the equatorial vicinity. For this latter particle, it is of interest to note that, somewhat before $t=0.8 \mathrm{~s}$, the pitch angle rapidly increases up to $150^{\circ}$, reflecting substantial parallel acceleration before the large energization achieved upon equatorial crossing $(\mathrm{t} \approx 0.8 \mathrm{~s})$. As mentioned above, this parallel acceleration is due to the pronounced curvature of the $\boldsymbol{E} \times \boldsymbol{B}$ drift path in the vicinity of the $Z=0$ plane (see Fig. $2 \mathrm{a}$ ). Indeed, in the absence of the parallel electric field and neglecting the effect of the gravitational acceleration, the parallel equation of motion of the guiding center is (e.g. Northrop, 1963):

$\frac{d V_{/ /}}{d t}=\mathbf{V}_{E} \quad \cdot\left[V_{/ /} \frac{\partial \boldsymbol{b}}{\partial s}+\mathbf{V}_{E} \cdot \nabla \mathbf{b}+\frac{\partial \boldsymbol{b}}{\partial t}\right]-\frac{\mu}{m} \frac{\partial B}{\partial s}$.

Here, $\mathbf{V}_{E}$ is the $\boldsymbol{E} \times \boldsymbol{B}$ drift velocity, $\mathbf{b}$, a unit vector in the $\mathbf{B}$ direction, $s$, the curvilinear abscissa along the field line, $\mu$, the particle magnetic moment and $m$, its mass. A similar equation (with the exception of the mirror force term) may be obtained in the full particle treatment (see, e.g. Sect. 3.2 of Delcourt et al., 1990), so that the following results do not depend upon whether the motion is adiabatic or not. The first term on the right-hand side of Eq. (4) is the parallel acceleration due to magnetic field line curvature (associated with the usual curvature drift), whereas the second term describes the acceleration due to the curvature of the $\boldsymbol{E} \times \boldsymbol{B}$ drift path.

In Fig. 5, these two curvature-related terms have been computed along selected orbits of Fig. 4. These two terms are shown as a function of instantaneous latitude until the first equatorial crossing (bottom panel), together with the net parallel speed (top panel). Consistently with the flow pattern displayed in Fig. 2, it is apparent from Fig. 5 that, immediately after the dipolarization onset, the test electrons are subjected to a prominent parallel acceleration and likely jetted toward the $Z=0$ plane. Most notably, it can be seen that, with the exception of the particle launched from the lowest latitude (red profile), the acceleration due to the curvature of the $\boldsymbol{E} \times \boldsymbol{B}$ drift paths (solid lines) largely exceeds that due to the magnetic field line curvature (dashed lines) until the very vicinity of the equator. At this point, the $\boldsymbol{E} \times \boldsymbol{B}$ drift path essentially points toward the planet and exhibits a negligible curvature, so that the associated centrifugal acceleration vanishes. Conversely, as will be made apparent in the following Fig. 6, this prominent equatorward centrifugal effect may lead to parallel deceleration and low-latitude mirroring for particles leaving the $Z=0$ plane, an effect that was referred to as "centrifugal trapping" (Delcourt et al., 1995).

Returning to Fig. 4, it can be seen that the $\boldsymbol{E} \times \boldsymbol{B}$ related centrifugal acceleration is substantial for particles launched 

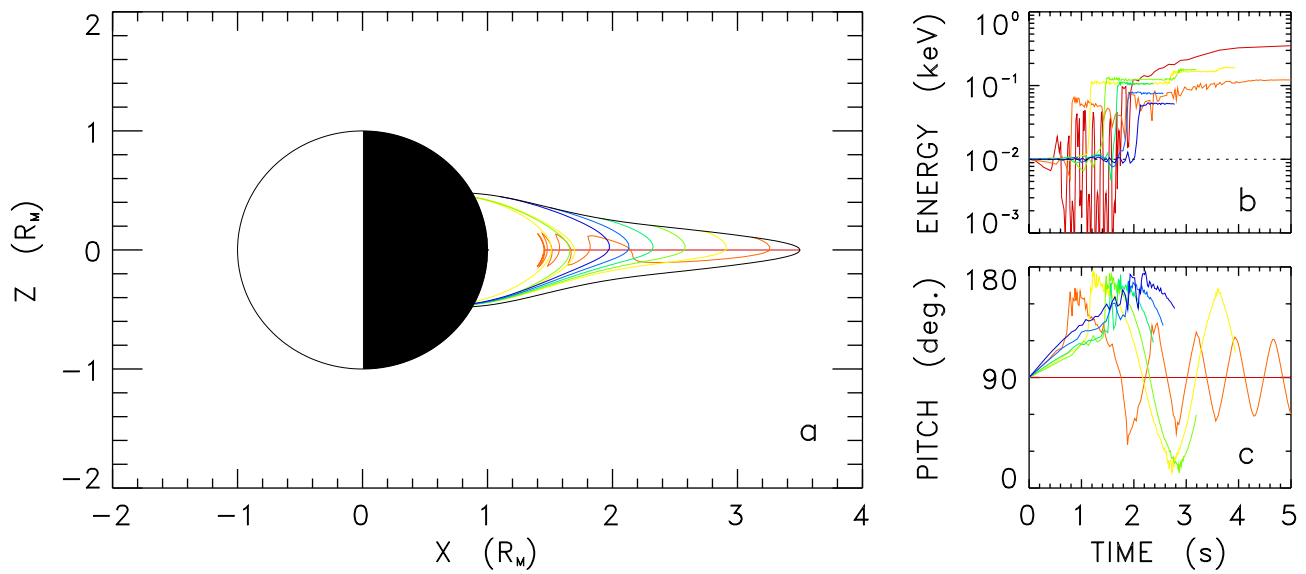

Fig. 4. Example of electron orbits for the model dipolarization shown in Fig. 2: (a) trajectory projections in the noon-midnight plane, (b) energy and (c) pitch angle versus time. The initial energy and pitch angle of the test electrons are set to $10 \mathrm{eV}$ and $90^{\circ}$, respectively. As in Fig. 2, the different colors correspond to distinct initial positions along the field line.
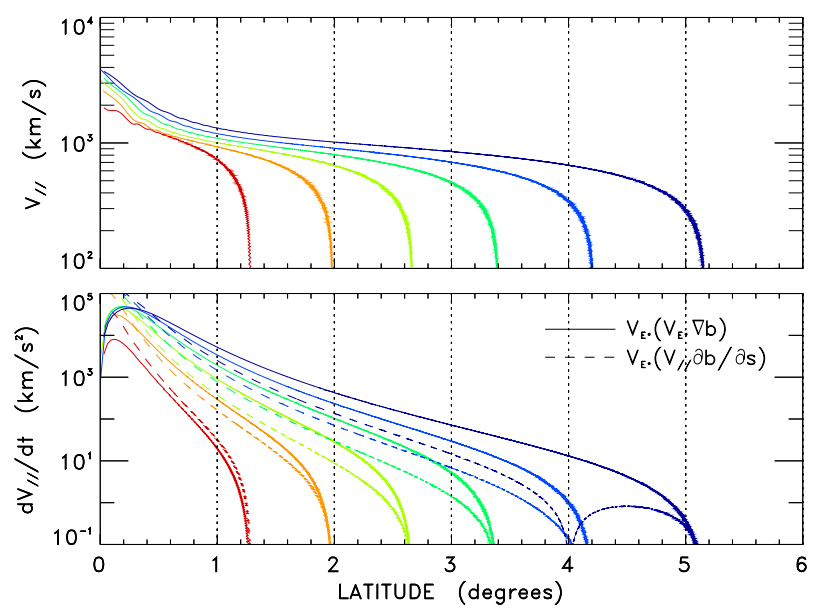

Fig. 5. (Top) Parallel speed and (bottom) parallel acceleration versus latitude for selected electron orbits in Fig. 4. In the bottom panel, dashed and solid lines correspond to accelerations due to magnetic field line curvature and to $\boldsymbol{E} \times \boldsymbol{B}$ drift path curvature, respectively (see Eq. 4).

from low initial latitudes (e.g. red, yellow and green trajectories) and that it is less pronounced at high initial latitudes, as expected from a locally weaker curvature of the $\boldsymbol{E} \times \boldsymbol{B}$ drift path (see Fig. 2). On the other hand, it is apparent from Fig. 4 that some of the test electrons that are initialized with $90^{\circ}$ pitch angle (or, equivalently, that have highaltitude mirror points) may experience a parallel acceleration that is large enough to lower the mirror point down to $1 \mathrm{R}_{M}$ radial distance (viz., assuming magnetic moment conservation, $B_{\text {final }}=B_{\text {initial }}\left[E_{\text {final }} / E_{\text {initial }}\right]$, where $B$ and $E$ denote the magnetic field at mirror point and the particle energy, respectively); hence, the precipitation of these electrons in the course of the dipolarization process.
A more general view of the electron dynamics and energy gain during dipolarization may be obtained from Fig. 6 which shows the results of trajectory calculations considering different initial energies, namely $10 \mathrm{eV}$ (blue profile) and $100 \mathrm{eV}$ (red) in the left panels, as well as $1 \mathrm{keV}$ (blue) and $10 \mathrm{keV}$ (red) in the right panels. Here, the test electrons have been launched from various latitudes (by steps of $0.2^{\circ}$ ) along the field line shown in Fig. 2, with an equatorial pitch angle (assuming magnetic moment conservation) of $10^{\circ}$. We also assumed that the test electrons initially travel from south to north by taking initial pitch angles smaller than $90^{\circ}$. Equivalently, the different initial latitudes in Fig. 6 correspond to different bounce phases for electrons with given initial energy. From top to bottom, the different panels in Fig. 6 show the following final parameters: radial distance, time, energy, magnetic moment (normalized to the initial value), and minimum $\kappa$ parameter encountered along the orbit (note that the particles may intercept the equatorial plane several times in the course of the dipolarization; hence, a series of $\kappa$ values).

In the top row of panels in Fig. 6, it is apparent that a number of test electrons precipitate onto the planet's surface $\left(\mathrm{R}_{\mathrm{final}}=1 \mathrm{R}_{M}\right)$ during dipolarization. In the second row of panels, this translates as times of flight smaller than $5 \mathrm{~s}$. Still, it can be seen that, while this precipitation occurs in a somewhat uniform manner (from $\sim-20^{\circ}$ up to $\sim-5^{\circ}$ and from $\sim 10^{\circ}$ up to $\sim 20^{\circ}$ initial latitude) for the lowest energy electrons (left panels), prominent latitudinal fluctuations are obtained for high-energy ones (right panels). The reason for this may be found in the bottom panels of Fig. 6 that show the minimum $\kappa$ parameter. Here, high-energy electrons have $\kappa$ well below unity and may be subjected to prominent magnetic moment scattering depending upon initial conditions; hence, the large fluctuations of the final radial distance. Note also that, because energetic electrons rapidly drift in azimuth, they may find themselves at a higher altitude than that expected from inward convection of the initial field line (dashed 

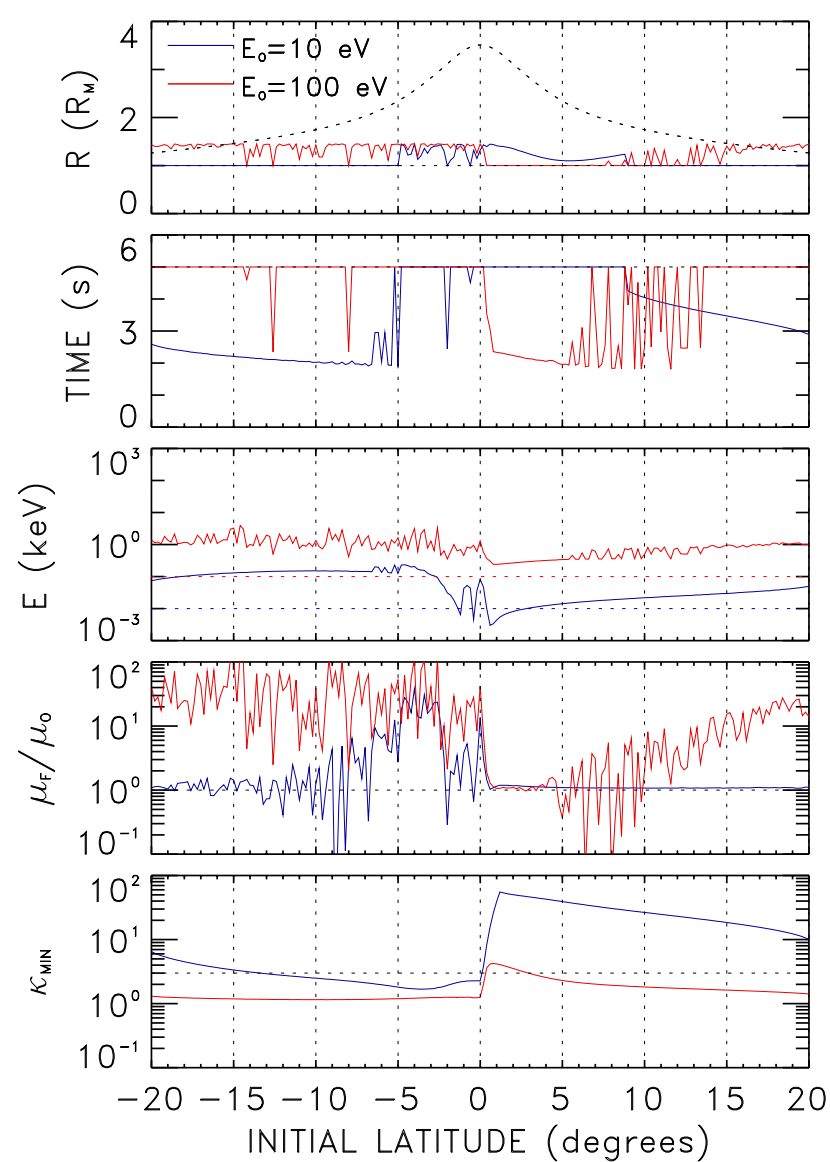
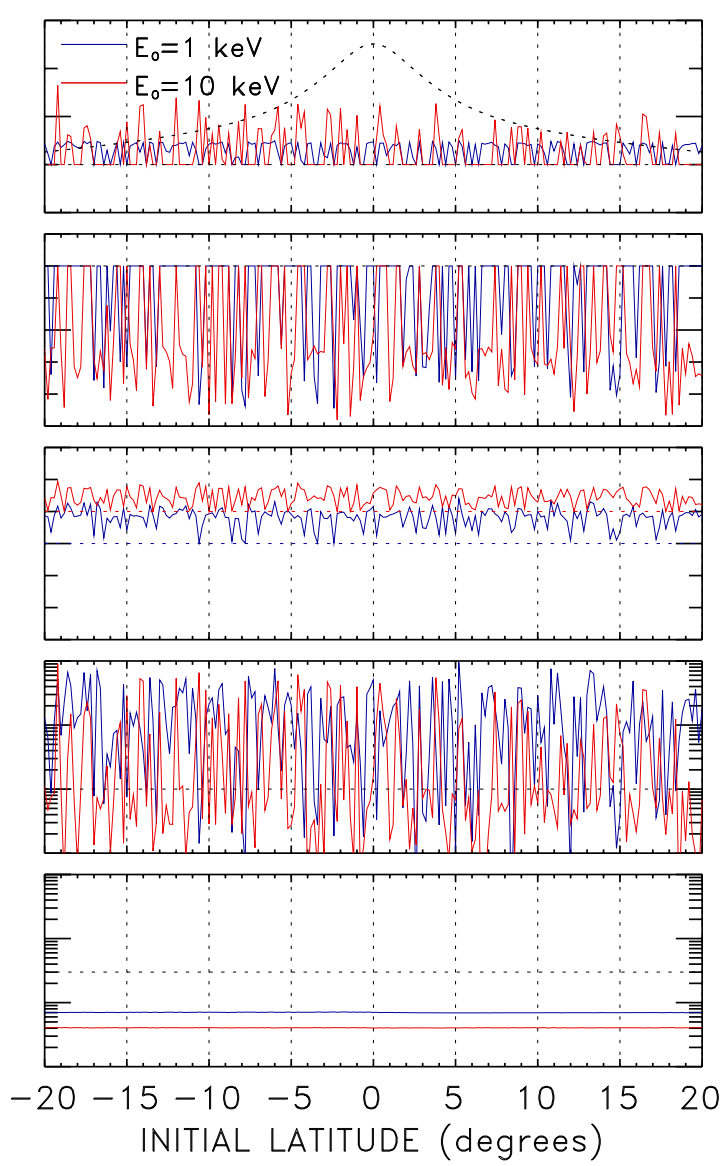

Fig. 6. (From top to bottom) Final radial distance, time of flight, energy, magnetic moment (normalized to the initial value), and minimum $\kappa$ parameter as a function of initial latitude. Left and right panels correspond to initial energies of $10-100 \mathrm{eV}$ and $1 \mathrm{keV}-10 \mathrm{keV}$, respectively, blue and red profiles relating to $(10 \mathrm{eV}, 1 \mathrm{keV})$ and $(100 \mathrm{eV}, 10 \mathrm{keV})$, respectively. All test electrons initially have the same pitch angle $\left(10^{\circ}\right)$ at equator, so that the different initial latitudes correspond to different bounce phases at the dipolarization onset.

line in top panels). In contrast, the bottom left panel of Fig. 6 indicates that the lowest energy electrons generally have $\kappa$ of the order of 3 or above, so that these particles behave adiabatically during dipolarization; hence, the smooth precipitation profile obtained in the top left panel (in this latter case, precipitation is due to a large parallel energization, whereas, for high-energy electrons, it follows from magnetic moment damping). Only a small fraction of the low-energy electrons (viz., those launched between $-5^{\circ}$ and $0^{\circ}$ latitude) have $\kappa$ of the order of 2 and experience a substantial magnetic moment enhancement.

Even though most of the low-energy electrons behave in an adiabatic (magnetic moment conserving) manner, it can be seen in the third panel from the top in Fig. 6 that their net energization strongly depends upon initial latitude (or, equivalently, the initial bounce phase). This is due to the large bounce period of these particles with respect to the dipolarization time scale (see Fig. 3) and reflects the violation of their second adiabatic invariant. Specifically, keeping in mind that the test electrons are assumed to travel from south to north at onset, it is apparent that those launched below the equator and which intercept the $Z=0$ plane during dipolarization experience a large (by about a factor of 10) energization. In contrast, electrons initialized above the equator experience a weaker energization, and possibly a deceleration due to the $\boldsymbol{E} \times \boldsymbol{B}$ related centrifugal effect for $\sim 1^{\circ}$ initial latitude. Such a uniform variation with initial latitude is not obtained for high-energy electrons (right panels) since the first adiabatic invariant is not conserved. In this latter case, fluctuations of the energy gain depend upon the net change in magnetic moment.

Figure 6 illustrates how nonadiabatic behaviors due to both spatial and temporal field variations are entangled in a complex manner. These may be summarized as follows: unlike ions, the short-lived electric field considered here does not lead to violation of the first adiabatic invariant for electrons because of their small cyclotron period (temporal adiabaticity). However, the second adiabatic invariant may be violated for low-energy electrons that have bounce periods comparable to or larger than the dipolarization time scale. On the other hand, low-energy electrons have Larmor radii smaller than the field variation length scale $(\kappa>3)$, so that the stretching of the magnetic field lines does not lead to violation of the first adiabatic invariant (spatial adiabaticity). 


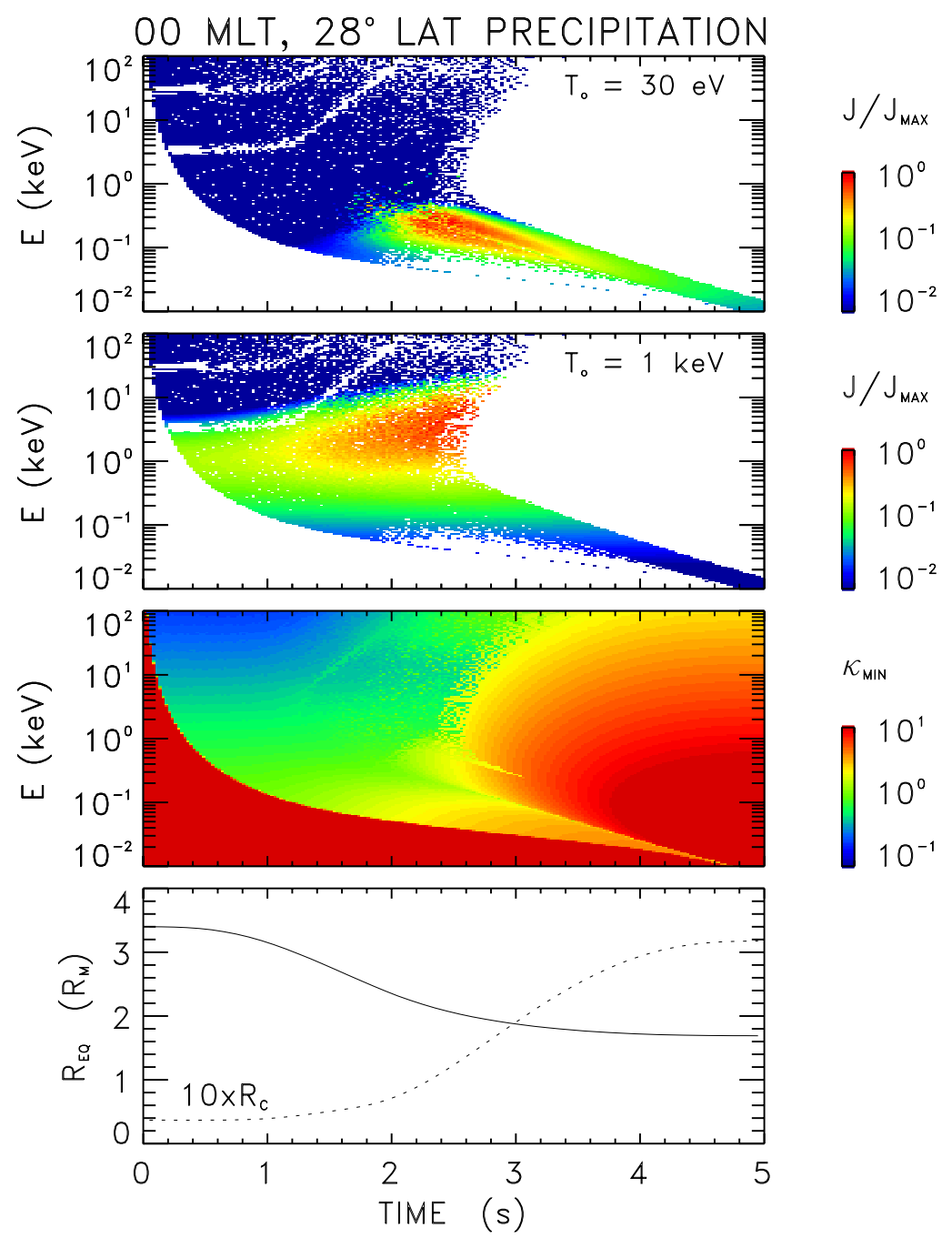

Fig. 7. (From top to bottom) Color-coded flux (normalized to the maximum value) for two different temperatures ( $30 \mathrm{eV}$ and $1 \mathrm{keV})$ of the initial Maxwellian distribution, and minimum $\kappa$ parameter as a function of time and energy. The lowermost shows the equatorial foot point of the field line anchored at $28^{\circ}$ latitude as a function of time. The curvature radius of this field line at the equator is also shown in a dotted line.

This is not the case, however, for high-energy electrons. Their large Larmor radii lead to $\kappa \approx 1$ or below, so that these latter particles possibly experience large magnetic moment changes.

\section{Injections of energetic electrons during dipolarization}

It was demonstrated above that, upon their injection toward the inner Hermean magnetosphere, electrons may be subjected to large energization under the effect of the transient electric field. In particular, it was shown that electrons may precipitate onto the planet's surface in the course of dipolarization, this precipitation resulting either from prominent parallel acceleration (for low-energy electrons that behave adiabatically) or from a decrease in the magnetic moment (for high-energy electrons that are nonadiabatically scattered upon crossing of the midplane).
In order to further examine this short-lived precipitation, we performed systematic trajectory calculations backward in time, for different energies from a given location (00 magnetic local time and $28^{\circ}$ latitude) at the planet's surface. These calculations were performed until the onset of dipolarization, at which point we considered that the electron distribution is an isotropic Maxwellian with an empty loss cone. Making use of the Liouville theorem that states conservation of the electron density in phase space, we then obtained the flux at a given time $t$ as: $J(t) / E(t) \propto \exp \left[-E_{\text {initial }} / k T_{\text {initial }}\right]$ (denoting by $E(t)$ the particle energy at time $t, E_{\text {initial }}$ its initial energy, and $T_{\text {initial }}$ the initial Maxwellian temperature).

The results of these calculations are shown in Fig. 7. The two top panels of this figure present the computed energy-time spectrograms considering two distinct temperatures of the initial distribution $(30 \mathrm{eV}$ and $1 \mathrm{keV})$, whereas the third panel from the top shows the minimum 

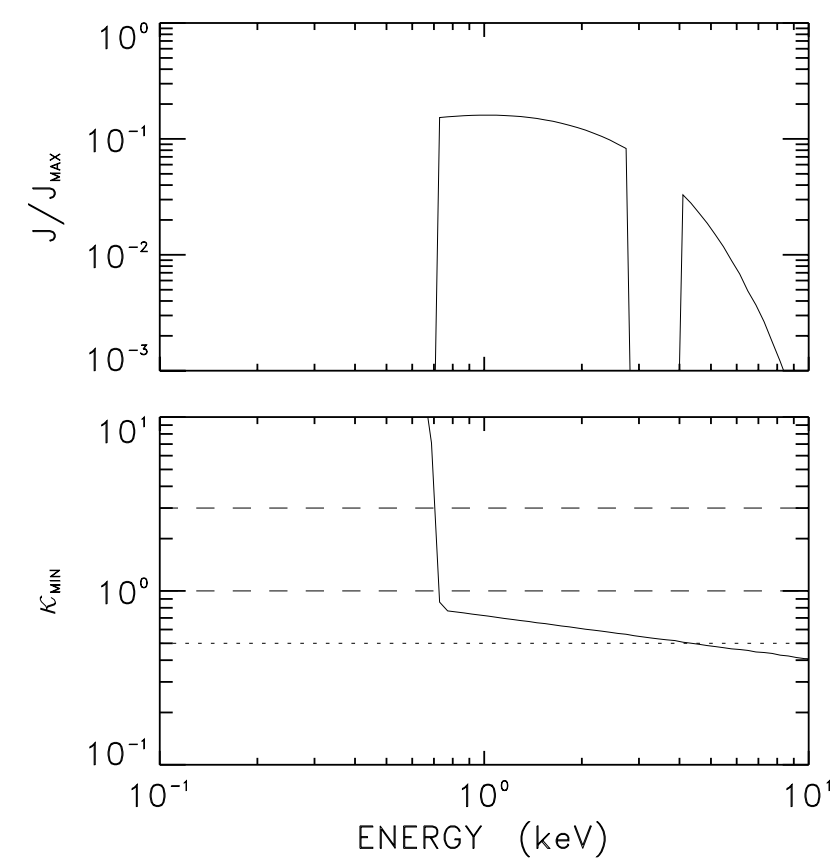

Fig. 8. (Top) Flux and (bottom) minimum $\kappa$ parameter as a function of energy at a given time $(t=0.4 \mathrm{~s})$ during the dipolarization process. This spectrum is obtained by assuming a $1-\mathrm{keV}$ temperature of the initial electron distribution (second panel from top in Fig. 7).

$\kappa$ parameter encountered during transport. Also, the bottom panel of Fig. 7 shows the time evolution of the equatorial foot of the field line anchored at the observation point. It is apparent from Fig. 7 that no electron precipitation occurs near the onset of dipolarization, since we assume the loss cone to be initially empty. On the other hand, as the dipolarization progresses and the equatorial foot of the field line gradually moves inward (bottom panel), it can be seen that substantial electron precipitation occurs with a clear cutoff at low energies, due to the time delay between the source and the planet's surface. This electron precipitation maximizes near half-collapse when the induced electric field reaches its peak magnitude. Here, stretching of the magnetic field line, combined with the intense electric field, maximizes the particle energization. On the whole, the bulk of the electron population experiences a net energization by about a factor of 5 within a few seconds. Subsequently (for $t>3 \mathrm{~s}$ ), the electron precipitation in Fig. 7 vanishes, except at the lowest energies where a clear time-of-flight dispersion can be seen. During this late stage of dipolarization, the magnetic field line exhibits a weaker curvature (dashed line in the bottom panel), that leads either to smaller parallel acceleration (hence, lesser lowering of the mirror point) or to less pronounced magnetic moment scattering (hence, negligible injection inside the loss cone).

Figure 7 displays significant electron precipitation induced by the dipolarization process, which may contribute to desorption of planetary material, such as $\mathrm{Na}$ or $\mathrm{K}$ atoms (e.g. Madey et al., 1998). This precipitation does not occur throughout the reconfiguration but within some fraction of it. Figure 7 also reveals that this precipitation seems to be absent within narrow energy bands. Insights into this latter effect may be obtained from Fig. 8 which shows the spectrum obtained at $t=0.4 \mathrm{~s}$ for $T_{\text {initial }}=1 \mathrm{keV}$ (second panel from top in Fig. 7). The top panel of Fig. 8 displays an electron flux that peaks near $1 \mathrm{keV}$ and rapidly decreases towards higher energies. A pronounced flux dropout is also noticeable at energies of the order of 3-4 keV.

The origin of this dropout can be understood by comparison with the bottom panel of Fig. 8 which shows the minimum $\kappa$ parameter as a function of energy. It can be seen in this panel that, whereas most of the electron spectrum covers $\kappa$ values up to 1 , the flux dropout corresponds to $\kappa$ of the order of 0.5. At this $\kappa$ value, Chen and Palmadesso (1986) demonstrated that a resonance occurs between the slow gyromotion about the magnetic field component normal to the midplane and the fast oscillation above and below this plane. As a result of this, particles experience little magnetic moment change and exhibit Speiser-type orbits (Speiser, 1965), a behavior referred to as quasi-adiabatic by Büchner and Zelenyi (1989). Because the magnetic moment is nearly unchanged in this regime, particles that precipitate after dipolarization initially are inside the loss cone. Since the loss cone is assumed to be empty in the present pre-dipolarization distribution, such particles are consequently absent in Fig. 8. In essence, the flux dropouts displayed here are thus similar to the modulation of ion distribution functions observed in the Earth's plasma sheet (e.g. Chen et al., 1990) and contains information on the magnetotail current sheet characteristics.

In order to investigate the injection of trapped electrons during the dipolarization process, we performed trajectory calculations similar to those in Fig. 7 but considering different final conditions, i.e. test electrons were traced backward in time for different energies from $X=1.5 \mathrm{R}_{M}$ (with $Y=Z=0$ ) and considering two distinct final pitch angles $\left(30^{\circ}\right.$ and $\left.80^{\circ}\right)$. The calculations were carried out until the onset of dipolarization, at which point we assumed the electron distribution to be an isotropic Maxwellian with an empty loss cone. The post-dipolarization electron flux at $1.5 \mathrm{R}_{M}$ was then calculated as described above.

The results of these calculations are shown in Fig. 9 which presents the computed energy-time spectrograms for two distinct initial temperatures (top panels), together with the color-coded initial MLT and minimum $\kappa$ encountered during transport (bottom panels). Note that, in contrast to Figs. 2, 4 and 7 , where the abscissa shows the time during the $5 \mathrm{~s}$ dipolarization, the abscissa in Fig. 9 shows the time measured from the end of the dipolarization (hence, its denomination as post-dipolarization time). Left and right panels in Fig. 9 correspond to the two distinct final pitch angles. Several features of interest may be noticed in this figure. First, in the upper left panel of Fig. 9, it can be seen that dipolarization leads to repeated flux enhancements on very short time scales (a few tenths of seconds) around $10-100 \mathrm{eV}$. As mentioned 


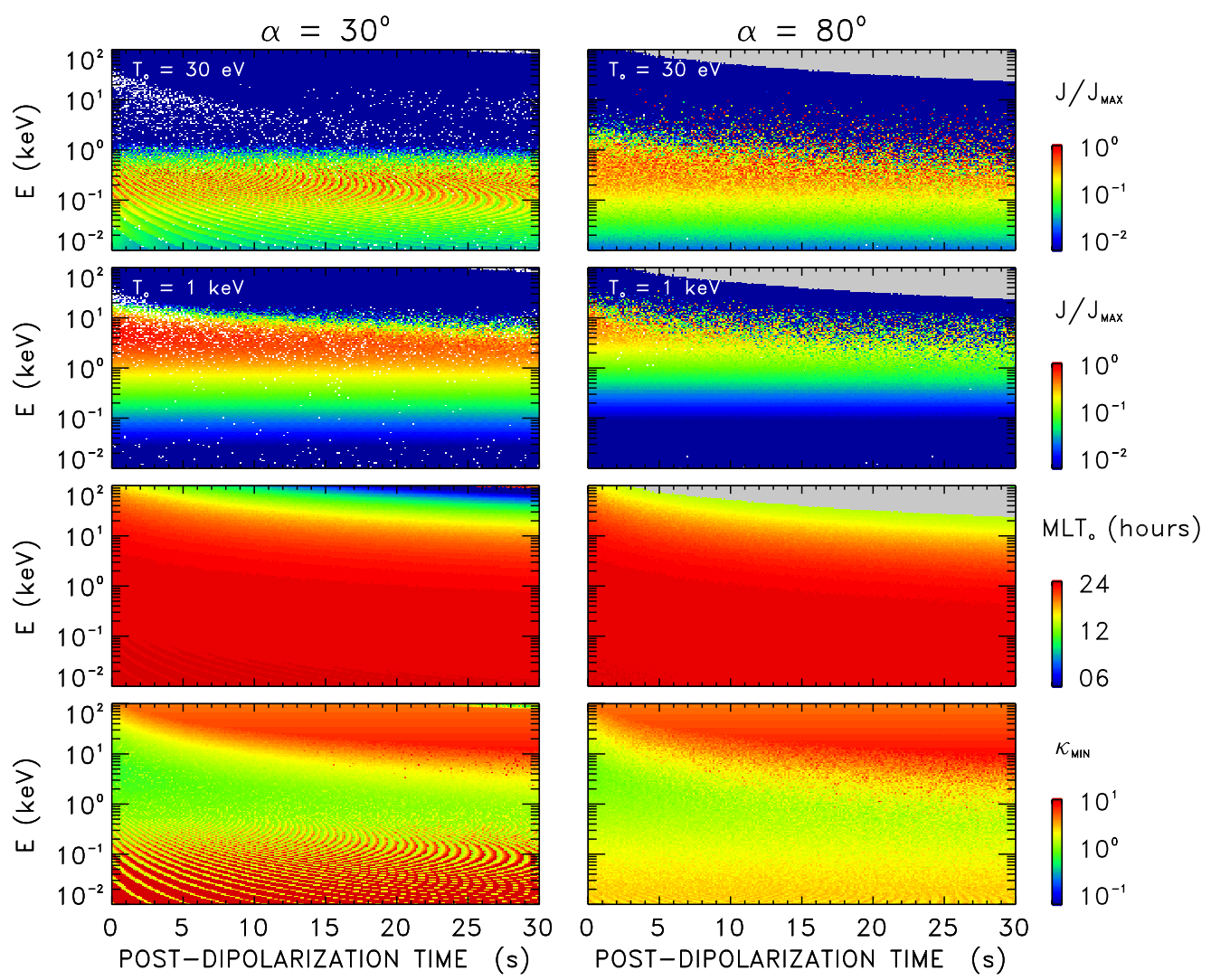

Fig. 9. (From top to bottom) Color-coded flux (normalized to the maximum value) for two different temperatures ( $30 \mathrm{eV}$ and $1 \mathrm{keV})$ of the initial Maxwellian distribution, initial MLT and minimum $\kappa$ parameter as a function of time and energy. Left and right panels correspond to two different post-dipolarization pitch angles at the equator $\left(30^{\circ}\right.$ and $80^{\circ}$, respectively). Grey bins correspond to particles intercepting the magnetopause.

above (see Fig. 6), these enhancements are due to clusters of accelerated electrons that bounce back and forth between the two hemispheres. They directly follow from the violation of the second adiabatic invariant and different parallel energization depending upon bounce phase.

As mentioned above, such bouncing structures are wellknown for plasma sheet ions in the inner Earth's magnetosphere (e.g. Quinn and McIlwain, 1979). At Mercury, however, due to small spatial and temporal scales, these structures are not obtained for ions which do not conserve their first adiabatic invariant but rather for low-energy electrons. In the bottom left panel of Fig. 9, it can be seen that the different energy gains experienced by the bouncing electrons translate as widely different $\kappa$ values, most of them being larger than 3, so that the electron motion indeed is adiabatic. Also, the upper left panel of Fig. 9 reveals that bouncing electron structures vanish above a few hundreds of $\mathrm{eV}$, consistently with a $\kappa$ parameter of the order of unity and consequent scattering of the electron magnetic moment. Finally, comparison of the top panels in Fig. 9 shows that such a structuring due to a bouncing motion is not obtained for large $\left(80^{\circ}\right)$ equatorial pitch angles.
On the other hand, for $1 \mathrm{keV}$ initial temperature (second panels from top in Fig. 9), prominent injections at energies up to a few tens of $\mathrm{keV}$ are noticeable, that decrease more rapidly in the perpendicular direction (right panel). At such energies, gradient drift becomes significant and particles may find themselves away from the midnight meridian at the dipolarization onset. This can be appreciated in the third panels from top in Fig. 9 which show the initial MLT of the test electrons. Here, a clear drift shell splitting effect is noticeable, i.e. conservation of both magnetic moment and longitudinal invariant is such that particles located at the same position after dipolarization but with different pitch angles circulate along different $L$-shells. As a matter of fact, it can be seen that energetic electrons with $80^{\circ}$ pitch angles originate from the frontside magnetopause (hence, the observed cutoff in the computed flux), whereas at $30^{\circ}$ pitch angle particles originate from the dawn sector. On the whole, Fig. 9 clearly demonstrates the possibility of large electron energization during substorm injection into the innermost magnetosphere. However, this figure does not exhibit short-lived $(\sim 6 \mathrm{~s})$ modulations similar to those reported by Mariner-10 (e.g. Christon et al., 1987), so that a convection surge alone does not seem sufficient to explain these latter observations. 


\section{Conclusions}

Test particle simulations using a rescaled time-dependent model of the Earth's magnetosphere were performed to investigate the dynamics of electrons during short-lived (several seconds) reconfigurations of Mercury's magnetotail. In a similar manner to the terrestrial magnetosphere, these simulations show that, during convection surges, electrons may be subjected to a rapid inward transport, as well as significant energization. They reveal that the electric field that is induced by dipolarization of the magnetic field lines may be responsible for transient electron precipitation onto Mercury's surface, either via prominent parallel energization or magnetic moment decrease. Prominent injections of trapped electrons are also obtained in the course of the convection surge. At relatively low energies (up to a few hundreds of eV), these injections are characterized by the formation of bouncing electron clusters due to violation of the second adiabatic invariant when the bounce period is larger than the field variation time scale. At Earth, such features are well-known for plasma sheet ions in the inner magnetotail. However, due to the small spatial and temporal scales of Mercury's magnetosphere, ions here do not conserve their first adiabatic invariant and such characteristic bouncing cluster signatures are obtained for electrons. At higher energies (several keVs and above), the pronounced stretching of the magnetic field lines in the Hermean magnetotail leads to a violation of the first adiabatic invariant of the electrons. Though a significant net energization may be obtained in the perpendicular direction, the present simulations that involve a single dipolarization event fail to reproduce the short-lived (6s) modulations of high-energy electrons observed by Mariner-10, which thus appear to follow from a different mechanism.

Acknowledgements. Part of this work was performed while D. C. Delcourt was residing at STEL, Toyokawa, Nagoya University (Japan).

Topical Editor T. Pulkkinen thanks two referees for their help in evaluating this paper.

\section{References}

Baker, D. N., Simpson, J. A., and Eraker, J. H.: A model of impulsive acceleration and transport of energetic particles in Mercury's magnetosphere, J. Geophys. Res., 91, 8742-8748, 1986.

Büchner, J. and Zelenyi, L. M.: Regular and chaotic charged particle motion in magnetotaillike field reversals: 1 . Basic theory of trapped motion, J. Geophys. Res., 94, 11 821-11 842, 1989.

Chen, J., Burkhart, G. R., and Huang, C. Y.: Observational signatures of nonlinear magnetotail particle dynamics, Geophys. Res. Lett., 17, 2237-2240, 1990.
Chen, J. and Palmadesso, P. J.: Chaos and nonlinear dynamics of single-particle orbits in magnetotaillike magnetic field, J. Geophys. Res., 91, 1499-1508, 1986.

Christon, S. P., Feynman, J., and Slavin, J. A.: Dynamic substorm injections: Similar magnetospheric phenomena at Earth and Mercury, AGU Monograph on "Magnetotail Physics", 393400, 1987.

Delcourt, D. C., Martin Jr., R. F., Sauvaud, J.-A., and Moore, T. E.: Centrifugal trapping in the magnetotail, Ann. Geophys., 13, 242-246, 1995,

SRef-ID: 1432-0576/ag/1995-13-242.

Delcourt, D. C., Sauvaud, J.-A., and Pedersen, A.: Dynamics of single-particle orbits during substorm expansion phase, J. Geophys. Res., 95, 20 853-20 865, 1990.

Eraker, J. H. and Simpson, J. A.: Acceleration of charged particles in Mercury's magnetosphere, J. Geophys. Res., 91, 9973-9993, 1986.

Heikkila, W. J. and Pellinen, R. J.: Localized induced electric field within the magnetotail, J. Geophys. Res., 82, 1610-1614, 1977.

Ip, W.-H.: Time-variable phenomena in the magnetosphere and exosphere of Mercury, Adv. Space Res., 19, 1615-1620, 1997.

Luhmann, J. G., Russell, C. T., and Tsyganenko, N. A.: Disturbances in Mercury's magnetosphere: Are the Mariner-10 "substorms" simply driven, J. Geophys. Res., 103, 9113-9120, 1998.

Madey, T. E., Yakshinskyi, B. V., Ageev, V. N., and Johnson, R. E.: Desorption of alkali atoms and ions from oxide surface: Relevance to origin of $\mathrm{Na}$ and $\mathrm{K}$ in atmospheres of Mercury and the Moon, J. Geophys. Res., 103, 5873-5888, 1998.

Mauk, B. H.: Quantitative modeling of the "convection surge" mechanism of ion acceleration, J. Geophys. Res., 91, $13423-$ $13431,1986$.

Mead, G. D. and Fairfield, D. H.: A quantitative magnetospheric model derived from spacecraft magnetometer data, J. Geophys. Res., 80, 523-534, 1975.

Mitchell, D. G., Brandt, P. C., Roelof, E. C., Hamilton, D. C., Retterer, K. C., and Mende, S.: Global imaging of $\mathrm{O}^{+}$from IMAGE/HENA, Space Sci. Rev., 109, 63-75, 2003.

Ness, N. F.: The magnetosphere of Mercury, in: Solar System Plasma Physics, edited by: Kennel, C. F., Lanzerotti, L. J., and Parker, E. N., North-Holland Publishing Company, 183206,1979 .

Northrop, T. G.: The adiabatic motion of charged particles, Wiley Interscience, New York, 1963.

Quinn, J. M. and McIlwain, C. E.: Bouncing ion clusters in the Earth's magnetosphere, J. Geophys. Res., 84, 7365-7370, 1979.

Russell, C. T. and Walker, R. J.: Flux transfer events at Mercury, J. Geophys. Res., 90, $11067-11$ 074, 1985.

Siscoe, G. L., Ness, N. F., and Yeates, C. M.: Substorms on Mercury, J. Geophys. Res., 80, 4359-4363, 1975.

Slavin, J. A.: Mercury's magnetosphere, Adv. Space Res., 33, 1859-1874, 2004.

Speiser, T. W.: Particle trajectory in model current sheets, 1, Analytical solutions, J. Geophys. Res., 70, 4219-4226, 1965.

Tsyganenko, N. A.: Magnetospheric magnetic field model with a warped tail current sheet, Planet. Space Sci., 37, 5-20, 1989. 